\title{
A generic travelling wave solution in dissipative laser cavity
}

\author{
BALDEEP KAUR* and SOUMENDU JANA \\ School of Physics and Materials Science, Thapar University, Patiala 147 004, India \\ ${ }^{*}$ Corresponding author. E-mail: baldeep@ thapar.edu
}

MS received 20 April 2015; revised 29 November 2015; accepted 16 December 2015; published online 9 September 2016

\begin{abstract}
A large family of cosh-Gaussian travelling wave solution of a complex Ginzburg-Landau equation (CGLE), that describes dissipative semiconductor laser cavity is derived. Using perturbation method, the stability region is identified. Bifurcation analysis is done by smoothly varying the cavity loss coefficient to provide insight of the system dynamics. He's variational method is adopted to obtain the standard sech-type and the notso-explored but promising cosh-Gaussian type, travelling wave solutions. For a given set of system parameters, only one sech solution is obtained, whereas several distinct solution points are derived for cosh-Gaussian case. These solutions yield a wide variety of travelling wave profiles, namely Gaussian, near-sech, flat-top and a coshGaussian with variable central dip. A split-step Fourier method and pseudospectral method have been used for direct numerical solution of the CGLE and travelling wave profiles identical to the analytical profiles have been obtained. We also identified the parametric zone that promises an extremely large family of cosh-Gaussian travelling wave solutions with tunable shape. This suggests that the cosh-Gaussian profile is quite generic and would be helpful for further theoretical as well as experimental investigation on pattern formation, pulse dynamics and localization in semiconductor laser cavity.
\end{abstract}

Keywords. Complex Ginzburg-Landau equation; dissipative system, stability analysis; He's variational method; cosh-Gaussian travelling wave solution.

\section{PACS Nos 42.65.-k; 42.65.Sf; 42.65.Tg}

\section{Introduction}

Self-sustaining localized structure that occurs in dissipative nonlinear systems, far from equilibrium, can be termed as dissipative soliton (DS) [1]. DSs are more wide-ranging than conservative solitons and occur in a larger variety of systems such as physical [2-4], chemical [5], mathematical [6], and biological systems [7]. They are also commonly observed in nature [8]. Since most of the practical systems are 'lossy', an external energy supply is required to keep the soliton 'alive' in such a dissipative system. On the contrary, conservative systems, which are comparatively rare in practice or 'oversimplified', do not require such energy supply. During the last decade, optical dissipative solitons (ODSs) attracted significant research attention. Cavity solitons (CSs) are a special type of ODS which can be excited inside a laser cavity. These are bright light spots on the dark background or conversely, dark spot on a homogeneous bright or grey background and appear on a plane transverse to the cavity axis [9]. CS can be assumed to be conventional propagating soliton bounded by the mirrors of the cavity, i.e., they are boundary localized along the propagating direction. Laser cavities of different types are available commercially, but the excitation of CS has been realized particularly in the vertical cavity surface emitting laser (VCSEL) because of its advantage over the other available devices [10]. The complex Ginzburg-Landau equation (CGLE) with cubic nonlinearity is a well-known model used for investigating pattern formation and dissipative optical solitons in VCSEL [11]. The generalized CGLE is such a classic equation that can be used for such purposes.

$$
\left(\frac{\partial}{\partial t}-\varepsilon\right) \psi=\left(1+i c_{1}\right) \frac{\partial^{2} \psi}{\partial x^{2}}-\left(1-i c_{3}\right)|\psi|^{2} \psi,
$$

where $\psi$ is the slowly-varying field envelope, $x$ is the spatial coordinate transverse to the cavity axis and $t$ is the cavity round trip time. To connect it with VCSEL, the coefficients $\varepsilon, c_{1}$ and $c_{3}$ should be considered as real-valued cavity parameters. Here, $\left(\partial_{t}-\varepsilon\right)$ describes 
the basic cavity dynamics. The system is considered to be simple and lossy, where $\varepsilon$ represents the cavity loss coefficient, scaled with respect to the evolution coordinate $t$. The term $\left(1+i c_{1}\right) \partial_{x x}$ describes the spatial coupling, wherein the real part represents diffusive coupling and imaginary part represents diffractive coupling. The last term $\left(1-i c_{3}\right)|\psi|^{2} \psi$ is the nonlinearity-induced loss/gain term in the system. The real and imaginary parts of the term describe, respectively, cubic nonlinear loss and nonlinear gain/absorption processes depending upon its sign. Equation (1) transforms to real Ginzburg-Landau equation for $c_{1}=c_{3}=0$, resulting in the onset of stationary periodic solution or stationary bifurcations [12]. The CGLE can also be converted to NLSE that counts the conservative system by setting $\varepsilon=0$ and eliminating unity terms in second and third terms of eq. (1):

$$
\frac{\partial \psi}{\partial t}=i c_{1} \frac{\partial^{2} \psi}{\partial x^{2}}+i c_{3}|\psi|^{2} \psi
$$

The prototype CGLE model described by eq. (1) has wide applications in a variety of physical phenomena starting from superconductivity to Bose-Einstein condensation [13]. Here, some primary references are brought forward. The study of travelling wave (TW) solution [14], spatiotemporal intermittency regime of NozakiBekki holes [11] and Benjamin-Feir stable region [15], localized TW solution [14,16], controlling of TW with feedback [17], turbulence in electromagnetic fields [18] and instability in nonlinear chemical kinetics [19] are to cite but a few. It is also sought after in superfluidity, second-order phase transitions, Rayleigh-Bénard convection [20]. The CGLE is also a commonly chosen model for pattern-forming systems and supports a variety of coherent and complex patterns [21,22]. This classic equation has been investigated to find localized structure in spatially extended non-equilibrium systems [21]. Several path-breaking experiments have been performed in optical systems that can be described by eq. (1) and its variants. Cavity soliton lasers, which can be driven externally through holding beam or self-driven with the help of feedback can host self-localized ODS. Such ODS, more particularly, CSs have been realized experimentally $[16,23,24]$. CS in self-driven system has been obtained by providing frequency-selective feedback to the cavity using Bragg grating [24-26].

A true CS can be envisaged as localized TW solutions. In a VCSEL coupled with feedback, many tilted TW can be excited [16]. They coexist, in some parametric region only, with the stable trivial solutions that correspond to the laser 'off' state. The tilted TWs interact with each other. The introduction of an annular spatial filter into the feedback path leads to a single dominant localized TW. Such localized structures are the basic requirement for CS generation. In the present investigation, we derived the TW solution of the CGLE, i.e., the primary step of the aforesaid mechanism of CS generation. However, our TW solution profile is new for such a system. Most of the theoretical works used bell shaped, i.e., sech or Gaussian profiles as an ansatz of the CGLE to start with. Moreover, different types of solutions have been reported for the generalized CGLE. In addition to stable stationary solutions, exact periodic and blow-up solutions have been derived using the homogeneous balance principle and Jacobi elliptic function. Peculiar results like periodic kink wave solutions, have been reported in ref. [27]. Depending upon the system dynamics and boundary conditions, types of periodic, quasiperiodic and chaotic solutions have been obtained for both the cubic [28] and cubic-quintic CGLE $[14,21,29]$. For a similar CGLE as described in eq. (1), but with cubic-quintic nonlinearity, kink and antikink stable bound states of solitons have been obtained analytically [30,31]. Stable vortical solitons for such quintic CGLE with radially inhomogeneous losses have been derived numerically [32]. Recently, triggered fronts of the CGLE have been reported in ref. [33].

In this paper, we determine a large family of coshGaussian TW solution of eq. (1). To the best of our knowledge, this is the first time that cosh-Gaussian profile has been used in the study of VCSEL, as well as of eq. (1). The cosh-Gaussian ansatz is found to be more generic as it gives rise to a number of different profiles, e.g., Gaussian, near-sech, flat-top and a typical double humped cosh-Gaussian profile with varying central dip, for different parametric values. This seems to be more interesting in comparison to the common sech profile for the spontaneous emergence and tunability of self-organized structures. Also, this profile promises more intriguing spatiotemporal patterns in spatially extended systems. Equation (1) is non-integrable by means of inverse scattering transform method, and hence no general exact solution is possible. However, a particular exact solution can be obtained [17,34,35]. Analytical approaches like Painlevé analysis and its variant [36] and Hirota bilinear method [37] have been employed to solve the CGLE. Soliton solutions of discrete CGLE have been obtained using extended hyperbolic function approach [38]. Besides, separation method has been found to be a very strong tool to solve such CGLE [30]. Approximate analytical method, such as variational method has been successfully used to 
find the dynamics of CGLE system [32,39]. Numerical solution of such CGLE mostly relies on CrankNicholson method. Time splitting spectral method is another strong contender [39,40].

In the present investigation, He's variational method [41] is adopted to find the sech and cosh-Gaussian TW solutions of CGLE. We have identified a parametric region of stable solution for the system. Bifurcation analysis is done to study the nature of the solution and to get better insight of the system. To validate the analytical results, split-step Fourier method, pseudospectral method and ETD2 exponential time-stepping method have been used. The versatile cosh-Gaussian TW thus obtained can be further used for the realization of CS in semiconductor laser cavity, which will be reported in the forthcoming communication.

This paper is organized as follows: $\$ 2$ consists of the stability analysis of the system under study. We present a brief bifurcation study in $\S 3$ for the totality of the investigation. In $\S 4$, the CGLE has been solved following He's variational method and standard sech as well as non-standard cosh-Gaussian TW solutions are obtained. Both analytical as well as numerical results are discussed in $\S 5$, which is followed by a conclusion in $\S 6$.

\section{Stability analysis}

One of the important and necessary exercises for studying a dynamical system is its stability analysis. A number of established mathematical techniques are available to analyse the stability criteria of a dynamical system. Lyapunov exponent's method [42], perturbation method [43], Eckhaus instability criteria [44] and Benjamin-Feir-Newell instability criteria [13], are a few to be listed. The system described by eq. (1) is nonconservative, and therefore, perturbative technique can be applied to the system in order to determine the stable and unstable zones in the parametric space. In order to obtain the homogeneous and steady state for the system, the following conditions must be satisfied:

$\frac{\partial^{2} \psi}{\partial x^{2}}=0 \quad$ and $\quad \psi=E_{0}$

assuming $E_{0}=\sqrt{I_{0}} \mathrm{e}^{i \omega t}$, where $I_{0}$ is the intensity and $\omega$ is the frequency of oscillation of the TW solution. Applying the conditions, stated in eq. (3) to eq. (1), we get

$i \omega=\varepsilon-\left(1-i c_{3}\right) I_{0}$.
On comparing real and imaginary parts, the following results are derived: (i) frequency of oscillation for the system $\omega=c_{3} \varepsilon$ and (ii) the intensity of the TW profile $I_{0}=\varepsilon$. To carry the study forward, perturbative analysis is applied to the system. The TW solutions are very useful in the starting hypothesis of perturbation theory or stability analysis [45]. The perturbed solution for the system described in eq. (1) can be written in the form: $\psi=E_{0}+\delta E \mathrm{e}^{i q x}$, where $\delta E$ is the amplitude of perturbed function and $q$ is the propagation constant of the TW. Substitution of the perturbed TW solution in eq. (1) results in

$$
\begin{aligned}
\frac{\partial}{\partial t}\left(\delta E \mathrm{e}^{i q x}\right)= & \varepsilon \delta E \mathrm{e}^{i q x}-q^{2}\left(1+i c_{1}\right) \delta E \mathrm{e}^{i q x} \\
& -\left(E_{0}^{2} \delta E^{*} \mathrm{e}^{-i q x}+2 I_{0} \delta E \mathrm{e}^{i q x}\right) \\
& +i c_{3}\left(E_{0}^{2} \delta E^{*} \mathrm{e}^{-i q x}+2 I_{0} \delta E \mathrm{e}^{i q x}\right) .
\end{aligned}
$$

Assuming $Q=\delta E \mathrm{e}^{i q x}$ and its complex conjugate $Q^{*}=\delta E^{*} \mathrm{e}^{-i q x}$ in eq. (5), we get

$$
\begin{aligned}
\frac{\partial Q}{\partial t}= & \varepsilon Q-q^{2}\left(1+i c_{1}\right) Q-\left(E_{0}^{2} Q^{*}+2 I_{0} Q\right) \\
& +i c_{3}\left(E_{0}^{2} Q^{*}+2 I_{0} Q\right) .
\end{aligned}
$$

For steady state, the parameters assume the value: $E_{0}=I_{0}=0$. Therefore, eq. (6) takes the following form:

$\frac{\partial Q}{\partial t}=\left[\left(\varepsilon-q^{2}\right)-i c_{1} q^{2}\right] Q$.

Equation (7) concludes that the system would bifurcate with frequency $\left(-c_{1} q\right)$. For non-steady state, i.e., $E_{0} \neq 0, I_{0} \neq 0, E_{0}=\sqrt{I_{0}} \mathrm{e}^{i \omega t}$, eq. (6) can be written in the rearranged form:

$\frac{\partial Q}{\partial t}=A Q+B Q^{*}$

where

$A=\varepsilon-q^{2}\left(1+i c_{1}\right)+2 I_{0}+2 i c_{3} I_{0}$

and

$B=\left(-E_{0}^{2}+i c_{3} E_{0}^{2}\right)$.

The complex conjugate of eq. (8) is

$\frac{\partial Q^{*}}{\partial t}=A^{*} Q^{*}+B^{*} Q$,

where $A^{*}$ and $B^{*}$ are the complex conjugates of $A$ and $B$ respectively. Equations (8) and (9) can be written in the matrix form as follows:

$\frac{\partial}{\partial t}\left[\begin{array}{c}Q \\ Q^{*}\end{array}\right]=\left[\begin{array}{cc}A & B \\ B^{*} & A^{*}\end{array}\right]\left[\begin{array}{c}Q \\ Q^{*}\end{array}\right]$. 
The eigenvalue determinant for eq. (10) is given by

$$
\left|\begin{array}{cc}
A-\lambda & B \\
B^{*} & A^{*}-\lambda
\end{array}\right|=0,
$$

where $\lambda$ is the eigenvalue of the system. The characteristic equation corresponding to the determinant in eq. (11) reads as

$\lambda^{2}-2 \operatorname{Re}(A) \lambda+|A|^{2}-|B|^{2}=0$.

For real values of $\lambda$, the system would transit from instability (for positive values of $\lambda$ ) to stability (for negative values of $\lambda$ ). In the proximity of this bifurcation, i.e., for $|\lambda| \ll 1$, the term $\lambda^{2}$ in eq. (12) can be neglected. Thus, we obtain

$\lambda=\frac{|A|^{2}-|B|^{2}}{2 \operatorname{Re}(A)}$.

Substituting the values of $A$ and $B$ in eq. (13), the expression for $\lambda$ takes the form:

$\lambda=\frac{\left(3 \varepsilon-q^{2}\right)^{2}+\left(2 c_{3} \varepsilon-q^{2} c_{1}\right)^{2}-\varepsilon^{2}\left(1+c_{3}^{2}\right)}{2\left(3 \varepsilon-q^{2}\right)}$.

In figure 1, the parametric space for eigenvalue $\lambda$ is plotted. Three distinct regions are identified. Regions I and III correspond to the negative eigenvalues and hence represent the stable TW solutions, whereas, Regions II and IV correspond to positive eigenvalues, thus represent the unstable region in parametric space.

\section{Bifurcation analysis}

The CGLE (eq. (1)) can be justifiably derived as an approximation at the wake of an oscillatory instability in dissipative systems [13]. Instability of CGLE has been observed in a variety of systems, for instance, RayleighBenárd convection in binary mixtures, hydrothermal waves and electroconvection in nematic liquid crystals [46]. For a better insight of the stability of the solution, bifurcation analysis of dynamical system is in order. Equation (1) is a regular form to show a large class of bifurcation phenomena in a spatially extended dissipative system. Bifurcation analysis of a system similar to eq. (1) has been done in ref. [47]. However, that investigation has been carried out by eliminating the diffusion term. Our analysis includes the total equation. If the term $\lambda^{2}$ is not neglected in the quadratic equation, (eq. (12)), then it results in

$\lambda=\operatorname{Re} A \pm \sqrt{(\operatorname{Re}(A))^{2}-|A|^{2}+|B|^{2}}$.

Smooth variation of the parameter $\varepsilon$ gives rise to a bifurcation. To obtain bifurcation diagram, we choose

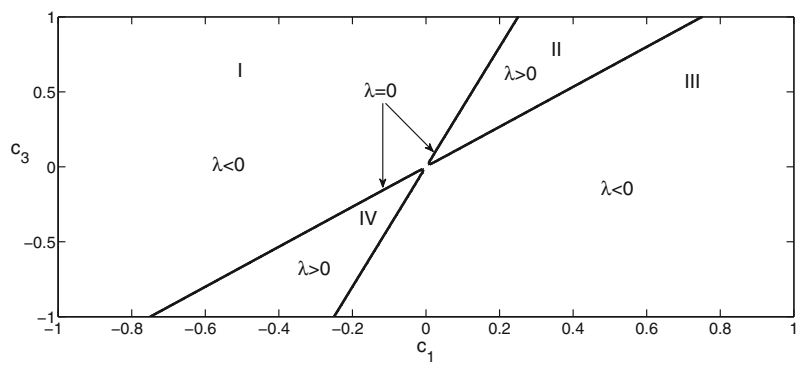

Figure 1. Stability region corresponding to eq. (13) for $\varepsilon=1$ and $q=2$. Regions I and III represent the stable region.

the values of the system parameters $c_{1}$ and $c_{3}$ from the stable zone of the stability plot (figure 1). For parametric values $c_{1}=0.2, c_{3}=0.8, I_{0}=\varepsilon$ and $q=4$, the system bifurcates at $\varepsilon=0$. Figure 2 shows the behaviour of dynamical system with the change in bifurcation parameter $\varepsilon$. Figure $2 \mathrm{i}$ corresponds to $\varepsilon>0$ that represents a stable spiral or attractor making the dynamical system stable. Figure 2 ii shows the unstable spiral for $\varepsilon<0$ that represents the unstable dynamical system. The behaviour of the system resembles the Hopf bifurcation [47]. The equilibrium point for the set of eqs (8) and (9) is $(0,0)$. This is represented by the red circle in figures $2 \mathrm{i}$ and $2 \mathrm{ii}$.

\section{Travelling wave solutions}

With the stability criteria for the system established, we proceed to find the TW solution of the system. Considering the TW hypothesis, the TW solution for the system can be written as

$\psi(x, t)=g(\xi) \mathrm{e}^{i(-k x+\omega t+\theta)}$,

where $g(\xi)$ is the profile function, the parameter $\xi=$ $x-v t, v$ is the TW velocity, $k$ is the wave number, $\omega$ is the frequency of the TW and $\theta$ is the phase constant. This TW solution is the natural extension of the stationary wave solution of the real equation corresponding to eq. (1). Substituting the value of $\psi(x, t)$ from eq. (16) in eq. (1), we obtain

$$
\begin{aligned}
& i\left(v g^{\prime}+\varepsilon g+g^{\prime \prime}-k^{2} g+2 c_{1} k g^{\prime}-g^{3}\right)+\omega g+2 k g^{\prime} \\
& \quad-c_{1} g^{\prime \prime}+c_{1} k^{2} g-c_{3} g^{3}=0
\end{aligned}
$$

where $g^{\prime}=\partial \psi / \partial \xi$ and $g^{\prime \prime}=\partial^{2} \psi / \partial \xi^{2}$. Separating the real and imaginary parts of eq. (17), we get

$\omega g+2 k g^{\prime}-c_{1} g^{\prime \prime}+c_{1} k^{2} g-c_{3} g^{3}=0$

and

$v g^{\prime}+\varepsilon g+g^{\prime \prime}-k^{2} g+2 c_{1} k g^{\prime}-g^{3}=0$. 


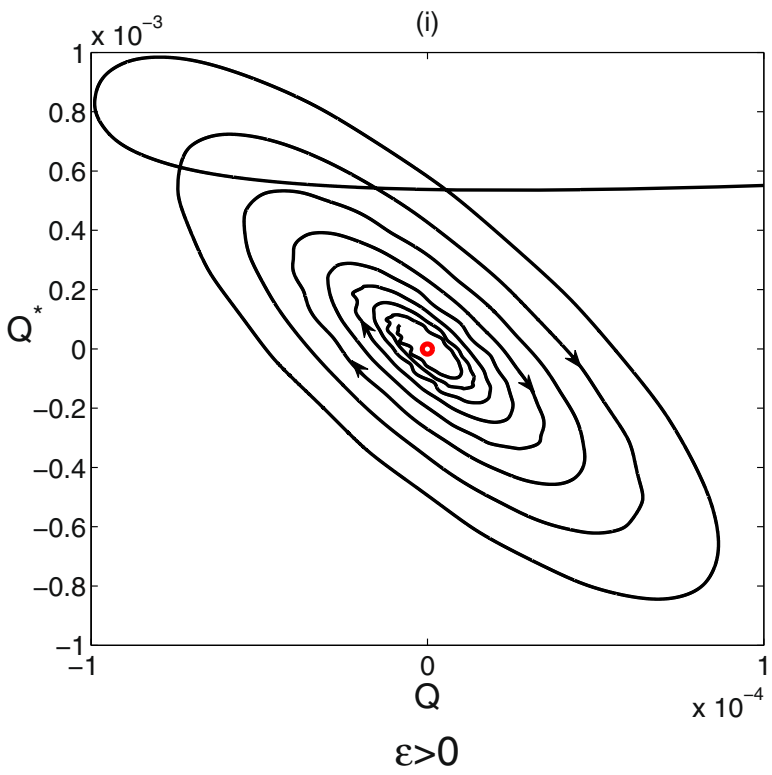

(ii)

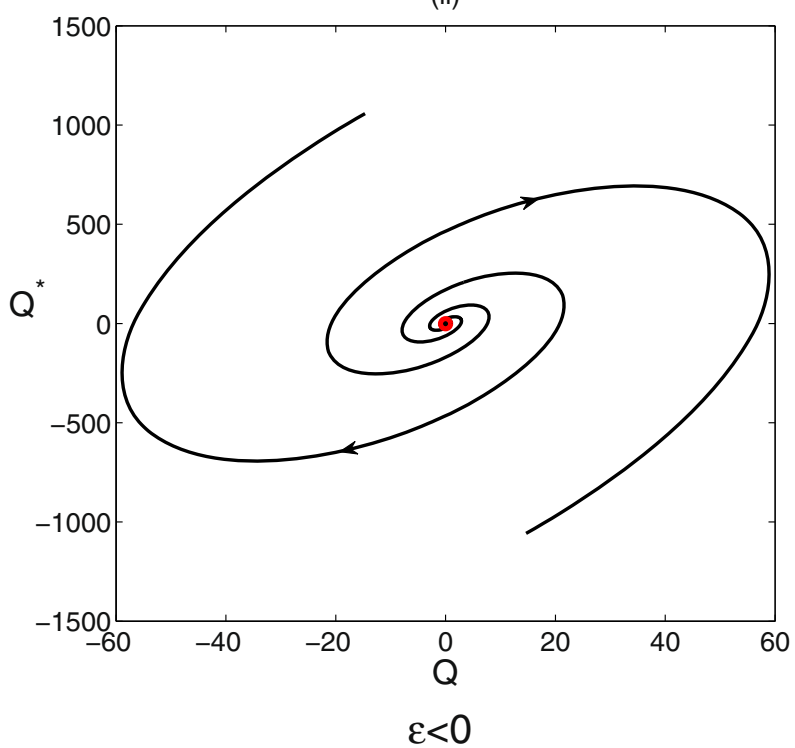

Figure 2. Bifurcation plot for the system of eqs (7) and (8). (i) The stable spiral for $\varepsilon>0$ and (ii) the unstable spiral for $\varepsilon<0$. $(0,0)$ (red circle) represents the equilibrium point for the system.

Equation (19) is then used to obtain the velocity of the TW using Laplace transform as follows:

Rearranging eq. (19), we get

$g^{\prime \prime}+\left(v+2 c_{1} k\right) g^{\prime}+\left(\varepsilon-k^{2}\right) g=g^{3}$,

with initial conditions $g(0)=g_{0}$ and $g^{\prime}(0)=0$. Here, $g_{0}$ is the initial value of the profile function $g(\xi)$ at $\xi=0$. The solution for nonlinear differential equations, like, eq. (20) is generally given by Volterra integral equation with initial value conditions as described in
Theorem 3.3 in ref. [48]. As in ref. [48], we consider two cases for $\lambda$, i.e., $\lambda>0$ and $\lambda<0$, where

$\lambda=\left(v+2 c_{1} k\right)^{2}-4\left(\varepsilon-k^{2}\right)$.

Case 1. For $\lambda>0$, the solution for eq. (20) is

$$
\begin{aligned}
g(\xi)= & k_{1} \mathrm{e}^{\alpha_{1} \xi}+k_{2} \mathrm{e}^{\alpha_{2} \xi} \\
& +\frac{1}{\sqrt{\lambda}} \int_{0}^{\xi}\left(\mathrm{e}^{\alpha_{1}(\xi-z)}-\mathrm{e}^{\alpha_{2}(\xi-z)}\right) g^{3}(z) \mathrm{d} z,
\end{aligned}
$$

where

$$
\begin{aligned}
& \alpha_{1}=\frac{\sqrt{\lambda}-\left(v+2 c_{1} k\right)}{2}, \\
& \alpha_{2}=\frac{-\sqrt{\lambda}-\left(v+2 c_{1} k\right)}{2}, \\
& k_{1}=\frac{\left(\sqrt{\lambda}-\left(v+2 c_{1} k\right)\right) g_{0}+2\left(v+2 c_{1} k\right)}{2 \sqrt{\lambda}}
\end{aligned}
$$

and

$k_{2}=\frac{\left(\sqrt{\lambda}-\left(v+2 c_{1} k\right)\right) g_{0}-2\left(v+2 c_{1} k\right)}{2 \sqrt{\lambda}}$.

Case 2. For $\lambda<0$, the solution for eq. (20) is

$g(\xi)=\mathrm{e}^{\alpha \xi}\left(b_{1} \cos \omega_{1} t+b_{2} \sin \omega_{1} t\right)$

$$
+\frac{2}{\sqrt{\lambda}} \int_{0}^{\xi} \mathrm{e}^{\alpha(\xi-z)} \sin \omega_{1}(\xi-z) g^{3}(z) \mathrm{d} z
$$

where

$$
\begin{aligned}
& \alpha=\frac{-\left(v+2 c_{1} k\right)}{2}, \\
& b_{1}=g_{0}, \\
& b_{2}=\frac{2 g_{0}\left(v+2 c_{1} k\right)}{\sqrt{\lambda}}
\end{aligned}
$$

and

$\omega_{1}=\frac{\sqrt{\lambda}}{2}$.

Further, four cases for the velocity $v$ of TW arise out of eq. (21), considering the conditions for $\lambda>0$ and $\lambda<0$. However, in our present discussion, we have considered only the case for $\lambda>0$ in order to keep the velocity positive. The limiting condition for velocity comes out to be

$v>-2 c_{1} k+2 \sqrt{\varepsilon-k^{2}}$.

Now, from eqs (18) and (19) we get

$$
\begin{aligned}
\omega g+ & 2 k\left(\frac{\left(g^{3}+\left(k^{2}-\varepsilon\right) g-g^{\prime \prime}\right)}{v+2 c_{1} k}\right) \\
& -c_{1} g^{\prime \prime}+c_{1} k^{2} g-c_{3} g^{3}=0 .
\end{aligned}
$$


Rearranging eq. (25), multiplying with $g^{\prime}$ and integrating over the whole space, we obtain

$$
\begin{aligned}
& \left(\omega+c_{1} k^{2}+\frac{2 k\left(k^{2}-\varepsilon\right)}{v+2 c_{1} k}\right) \frac{g^{2}}{2}+\left(\frac{2 k}{v+2 c_{1} k}-c_{3}\right) \frac{g^{4}}{4} \\
& -\left(c_{1}+\frac{2 k}{v+2 c_{1} k}\right) \frac{\left(g^{\prime}\right)^{2}}{2}=K_{1} .
\end{aligned}
$$

A new quantity $J$ is introduced, which can be defined as

$$
J=\int_{-\infty}^{\infty} K_{1} \mathrm{~d} \xi
$$

This physical quantity $J$ in conjugation with an appropriate profile function, gives rise to the TW solution. In literature, numerous profile functions have been discussed starting from the simple Gaussian to chirped Gaussian [1,49] and sinh-Gaussian [50]. In this work, the simplest sech $[30,51]$ and rarely used, but more generic, cosh-Gaussian profile functions [52] have been discussed.

\section{Case I. Standard sech TW ansatz}

We derived the standard sech TW solution of eq. (1) for the totality of the investigation and comparison with the cosh-Gaussian profile. We take the profile function as

$g(\xi)=S \operatorname{sech}(T \xi)$,

where $S$ represents the amplitude and $T$ represents the inverse width. Substituting eq. (28) in eq. (27) and integrating, we obtain

$$
\begin{aligned}
J_{1}= & \frac{S^{2}}{T}\left(\omega+c_{1} k^{2}+\frac{2 k\left(k^{2}-\varepsilon\right)}{v+2 c_{1} k}\right) \\
& +\frac{S^{4}}{3 T}\left(\frac{2 k}{v+2 c_{1} k}-c_{3}\right) \\
& -\frac{S^{2} T}{3}\left(c_{1}+\frac{2 k}{v+2 c_{1} k}\right) .
\end{aligned}
$$

In order to obtain the values of constant parameters $S$ and $T$, the principle of variation, i.e., $\partial J_{1} / \partial S=0$ and $\partial J_{1} / \partial T=0$ is applied. This results in the following conditions respectively:

$$
\begin{aligned}
& \frac{S}{T}\left(\omega+c_{1} k^{2}+\frac{2 k\left(k^{2}-\varepsilon\right)}{v+2 c_{1} k}\right)+\frac{2 S^{3}}{3 T}\left(\frac{2 k}{v+2 c_{1} k}-c_{3}\right) \\
& -\frac{S T}{3}\left(c_{1}+\frac{2 k}{v+2 c_{1} k}\right)=0
\end{aligned}
$$

and

$$
\begin{aligned}
& \frac{S^{2}}{T^{2}}\left(\omega+c_{1} k^{2}+\frac{2 k\left(k^{2}-\varepsilon\right)}{v+2 c_{1} k}\right)+\frac{S^{4}}{3 T^{2}}\left(\frac{2 k}{v+2 c_{1} k}-c_{3}\right) \\
& +\frac{S^{2}}{3}\left(c_{1}+\frac{2 k}{v+2 c_{1} k}\right)=0 .
\end{aligned}
$$

Upon solving eqs (30) and (31), thus result in

$S=\left[\frac{2\left(\omega+c_{1} k^{2}+\frac{2 k\left(k^{2}-\varepsilon\right)}{v+2 c_{1} k}\right)}{\left(c_{3}-\frac{2 k}{v+2 c_{1} k}\right)}\right]^{1 / 2}$

and

$T=\left[-\frac{\left(\omega+c_{1} k^{2}+\frac{2 k\left(k^{2}-\varepsilon\right)}{v+2 c_{1} k}\right)}{c_{1}+\frac{2 k}{v+2 c_{1} k}}\right]^{1 / 2}$.

The TW profile function assumed in eq. (28) and the conditions derived for parameters $S$ and $T$ in eq. (32), results in figure 3 . The values of the system parameters used are chosen from the stable zone of figure 1 and are given by $c_{1}=0.3, c_{3}=-0.8, \varepsilon=1, I_{0}=\varepsilon$, $\omega=c_{3}, k=0.3$ and $v=11.7279$. We also obtained similar TW profiles with other suitable points of the stable zone.

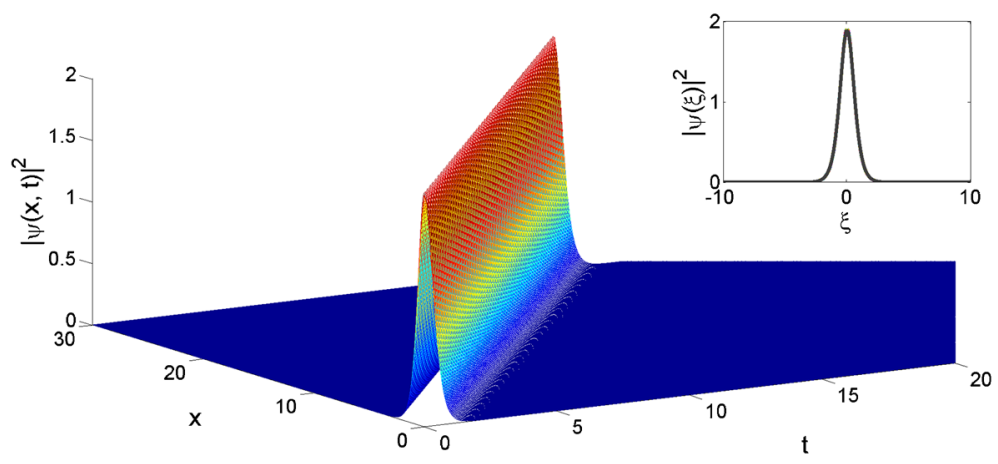

Figure 3. 3D Intensity profile for sech $\mathrm{TW}$ solution with $2 \mathrm{D}$ profile in the inset. 
Case II. Non-standard cosh-Gaussian TW ansatz

In this case, we propose the following unchirped coshGaussian ansatz as profile function:

$g(\xi)=P \cosh (Q \xi) \exp \left(-\xi^{2} / R^{2}\right)$,

where $P$ represents the amplitude, $Q$ is the cosh parameter and $R$ is the pulse width parameter of the profile. Such cosh-Gaussian TW is rarely adopted but quite generic in nature. Substituting eq. (33) in eq. (27) and integrating, we obtain the expression for physical quantity $J_{2}$ as

$$
\begin{aligned}
J_{2}=\sqrt{\pi} & {\left[\frac{\sqrt{2}}{8} a_{1} P^{2} R\left(1+\mathrm{e}^{\frac{Q^{2} R^{2}}{2}}\right)\right.} \\
& +\frac{a_{2}}{64} P^{4} R\left(\mathrm{e}^{Q^{2} R^{2}}+4 \mathrm{e}^{\frac{Q^{2} R^{2}}{4}}+3\right) \\
& \left.-\frac{\sqrt{2} a_{3}}{8} \frac{P^{2}}{R}\left(1-Q^{2} R^{2}+\mathrm{e}^{\frac{Q^{2} R^{2}}{2}}\right)\right],
\end{aligned}
$$

where

$$
\begin{aligned}
& a_{1}=\left(\omega+c_{1} k^{2}+\frac{2 k\left(k^{2}-\varepsilon\right)}{v+2 c_{1} k}\right), \\
& a_{2}=\left(\frac{2 k}{v+2 c_{1} k}-c_{3}\right)
\end{aligned}
$$

and

$a_{3}=\left(c_{1}+\frac{2 k}{v+2 c_{1} k}\right)$.

Applying the principle of variation to eq. (34), i.e., $\partial J_{2} / \partial P=0, \partial J_{2} / \partial Q=0$ and $\partial J_{2} / \partial R=0$, we obtain the following three equations respectively:

$$
\begin{aligned}
& \frac{\sqrt{2} a_{1}}{4} P R\left(1+\mathrm{e}^{\frac{Q^{2} R^{2}}{2}}\right)+\frac{a_{2}}{16} P^{3} R\left(\mathrm{e}^{Q^{2} R^{2}}+4 \mathrm{e}^{\frac{Q^{2} R^{2}}{4}}+3\right) \\
& -\frac{\sqrt{2} a_{3}}{4} \frac{P}{R}\left(1-Q^{2} R^{2}+\mathrm{e}^{\frac{Q^{2} R^{2}}{2}}\right)=0 \\
& \frac{\sqrt{2} a_{1}}{8} P^{2} R^{3} Q \mathrm{e}^{\frac{Q^{2} R^{2}}{2}}+\frac{a_{2}}{32} P^{4} R^{3} Q\left(\mathrm{e}^{Q^{2} R^{2}}+\mathrm{e}^{\frac{Q^{2} R^{2}}{4}}\right) \\
& -\frac{\sqrt{2} a_{3}}{8} P^{2} Q R\left(-2+\mathrm{e}^{\frac{Q^{2} R^{2}}{2}}\right)=0
\end{aligned}
$$

and

$$
\begin{aligned}
& \frac{\sqrt{2} a_{1}}{8} P^{2}\left(1+\mathrm{e}^{\frac{Q^{2} R^{2}}{2}}\right)+\frac{\sqrt{2} a_{1}}{8} P^{2} Q^{2} R^{2} \mathrm{e}^{\frac{Q^{2} R^{2}}{2}} \\
& +\frac{a_{2}}{64} P^{4}\left(\mathrm{e}^{Q^{2} R^{2}}+4 \mathrm{e}^{\frac{Q^{2} R^{2}}{4}}+3\right) \\
& +\frac{a_{2}}{32} P^{4} Q^{2} R^{2}\left(\mathrm{e}^{Q^{2} R^{2}}+\mathrm{e}^{\frac{Q^{2} R^{2}}{4}}\right) \\
& +\frac{\sqrt{2} a_{3}}{8} \frac{P^{2}}{R^{2}}\left(1-Q^{2} R^{2}+\mathrm{e}^{\frac{Q^{2} R^{2}}{2}}\right) \\
& -\frac{\sqrt{2} a_{3}}{8} P^{2} Q^{2}\left(-2+\mathrm{e}^{\frac{Q^{2} R^{2}}{2}}\right)=0 .
\end{aligned}
$$

Equations (35)-(37) are solved to determine the parameters $P, Q$ and $R$.

\section{Results and discussion}

It is very cumbersome to solve eqs (35)-(37) analytically. Subsequently, these equations are solved graphically. Different values of parameters generate different solution points, hence different nature of profiles are witnessed in each case. One of the possible cases is discussed in this study. Figure 4 depicts the first quadrant of solution plot for eqs (35)-(37) with variation in parameters $P$ and $Q$, keeping $R(=0.3)$ constant for parametric values $c_{1}=0.3, c_{3}=-0.8, \varepsilon=1, I_{0}=\varepsilon$, $\omega=c_{3}, k=0.3$ and $v=11.7279$. It may be noted that all the figures in this section, obtained from both analytical as well as numerical analysis, are plotted for system parameters that correspond to stable zone of figure 1. Red line corresponding to eq. (35) and black dotted line corresponding to eq. (36), intersect at eight different points, thus creating eight different solution points for the same width $R$. Inset provides the zoom-in view of the intersection points (c), (d), (e), (f), $(\mathrm{g})$ and $(\mathrm{h})$ marked in figure 4 . Each solution point generates different pulse profiles, providing a variety of profile shapes. Figure 5 i shows the intensity profile plots in 3D with 2D plot in figure 5ii for point (a) that corresponds to $P=2.7994$ and $Q=0$. Intensity profile in figure $5 \mathrm{i}$ is a purely Gaussian shape profile, closely matching the nature of standard sech profile shape. Figure 5iii is the 3D intensity profile for $P=1.9402$ and $Q=4.7740$ corresponding to the point (b) in figure 4 and the corresponding 2D profile shape shown in figure $5 \mathrm{iv}$. It is a flat-top profile. Typical cosh-Gaussian profile with $3 \mathrm{D}$ and cross-sectional $2 \mathrm{D}$ profile is plotted for the solution point (j) in figures $5 \mathrm{v}$ and $5 \mathrm{vi}$, respectively. Solution points (c)-(h) in figure 4 generate a similar pulse with a dip in between two equally 


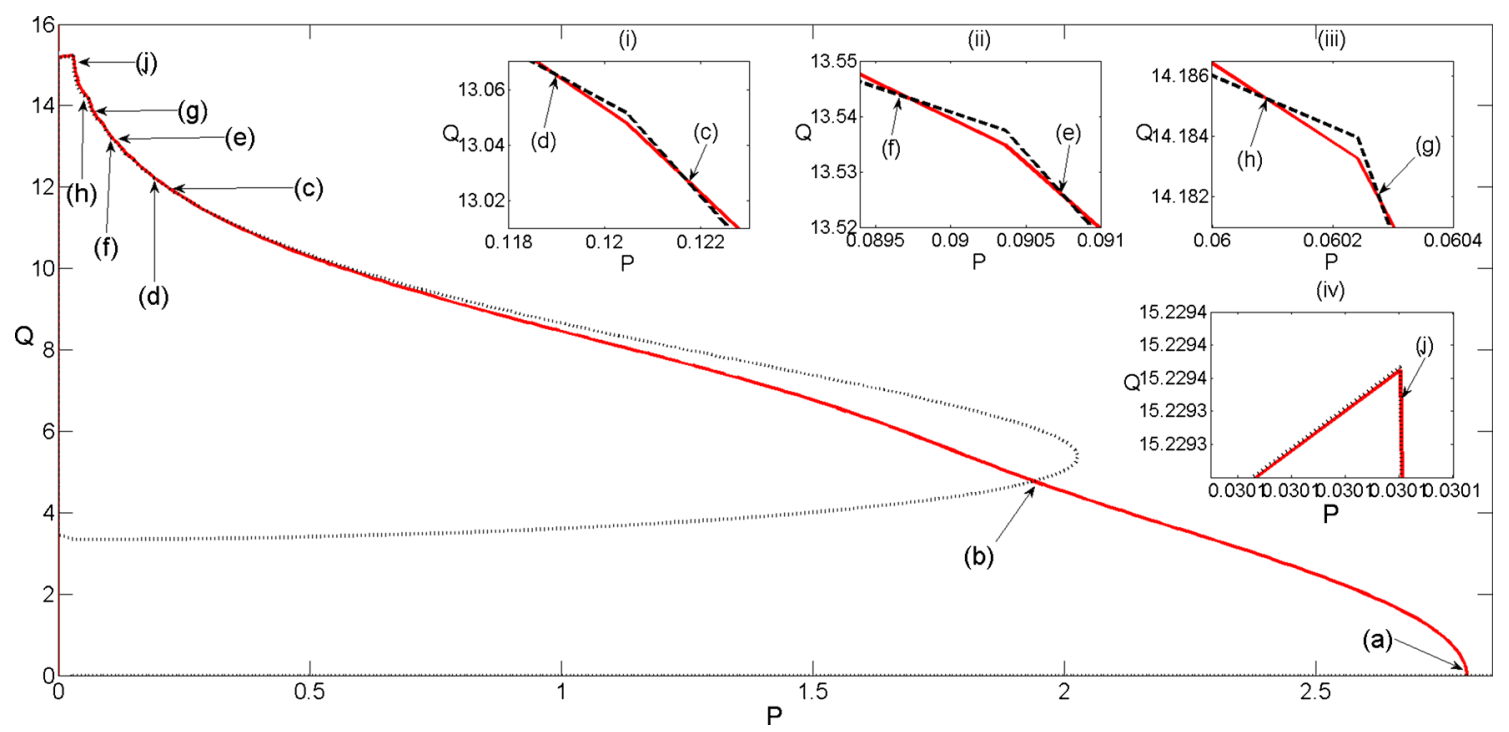

Figure 4. Parametric solution space plot for parameters $P$ and $Q$ in eqs (32) and (33) for constant $R(=0.3)$. Insets are zoom-in view of solution points marked in the main plot: (i) correspond to points (c) and (d), (ii) correspond to points (e) and (f), (iii) corresponds to points (g) and (h) and (iv) corresponds to point (j).

distributed maxima. These points represent the typical cosh-Gaussian nature of the profile. Solution point pairs $(\mathrm{c})((P, Q)=(0.1217,13.0282))$ and $(\mathrm{d})((P, Q)=$ $(0.0908,13.5247)),(\mathrm{e})((P, Q)=(0.0897,13.5435))$ and (f) $((P, Q)=(0.0603,14.1817))$, generate almost similar pulse profiles, with a dip nearly touching the base line (see figures 6i and 6ii, 6iii and 6iv). 2D profile plots of the next two solution points, i.e., $(\mathrm{g})((P$, $\mathrm{Q})=(0.0600,14.1859))$ and $(\mathrm{h})((P, Q)=(0.0301$,
15.2294)), are depicted in figures 6v and 6vi. As the value of $Q$ increases, the dip in between two humps flattens at the base. Each solution point shows similar nature of pulse profile with almost well separated peaks. It is interesting to note that as the value of coshGaussian parameter $Q$ increases, the central dip starts forming in the intensity profile. As the value of $P$ decreases and $Q$ increases, the dip tends to touch the $x$-axis with flattening bottom. The root mean square
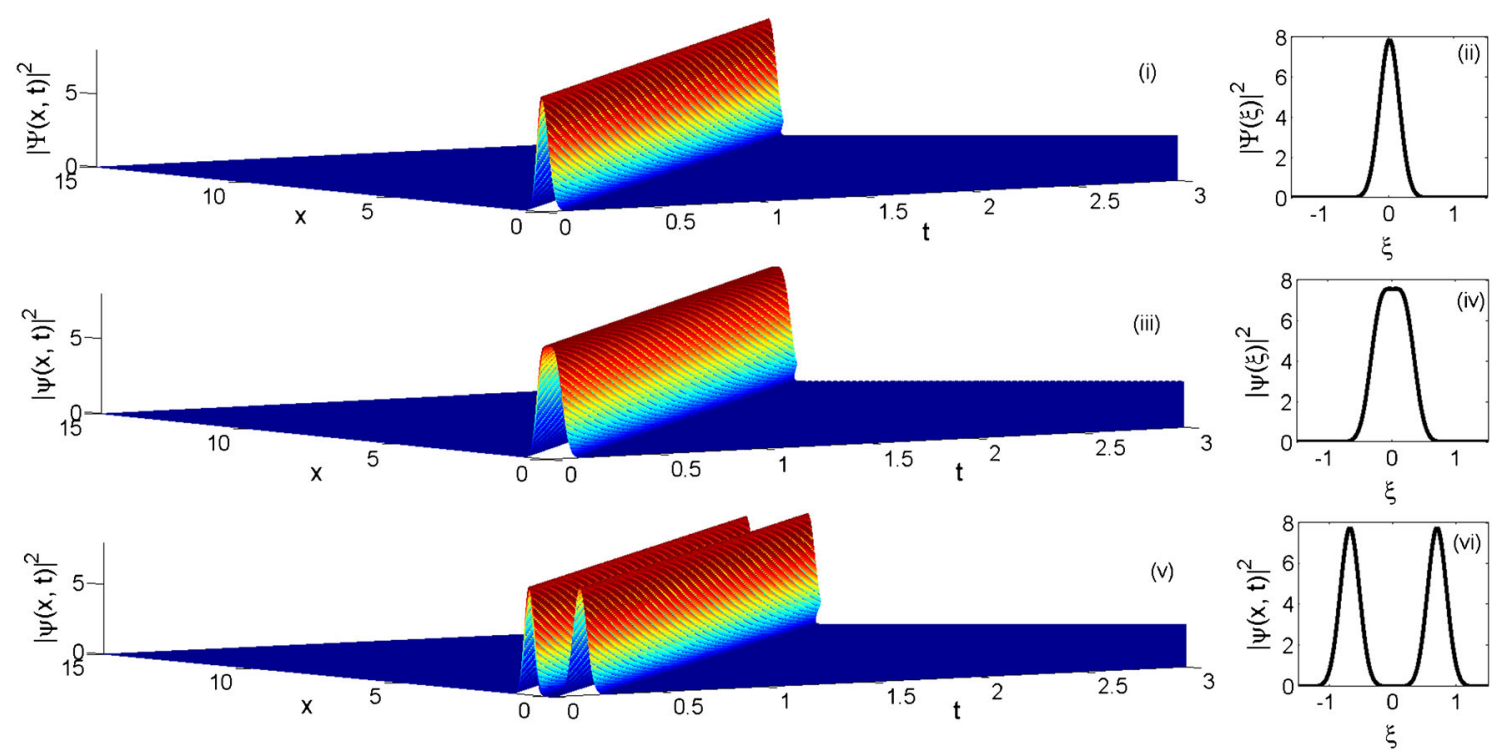

Figure 5. 3D intensity profile for (i) solution point (a), i.e., $(P, Q)=(2.7994,0)$, (iii) solution point (b), i.e., $(P, Q)=$ $(1.9402,4.7740)$, $(\mathbf{v})$ solution point $(\mathrm{j})$, i.e., $(P, Q)=(0.0301,15.2294)$. Respective $2 \mathrm{D}$ profiles are plotted in (ii), (iv) and (vi). 
(i)

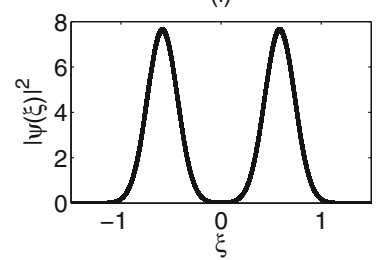

(iv)

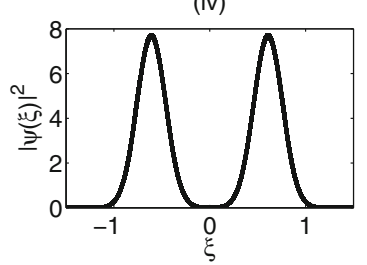

(ii)

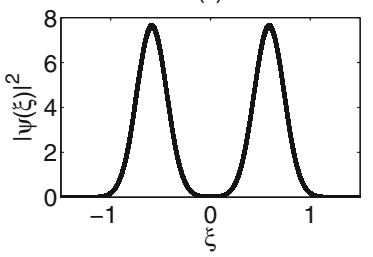

(v)

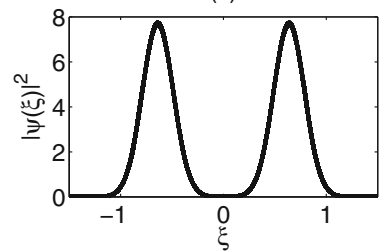

(iii)

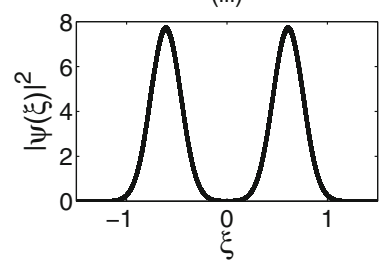

(vi)

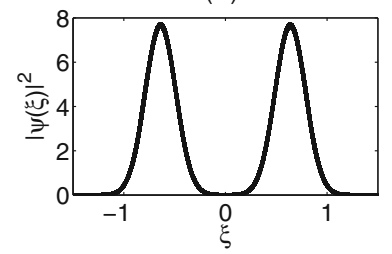

Figure 6. 2D intensity profiles corresponding to (i) solution point (c) $((P, Q)=(0.1217,13.0282))$, (ii) solution point (d) $((P, Q)=(0.1189,13.0664))$, (iii) solution point $(\mathrm{e})((P, Q)=(0.0908,13.5247))$, (iv) solution point $(\mathrm{f})((P, Q)=$ $(0.0897,13.5435)),(\mathbf{v})$ solution point $(\mathrm{g})((P, Q)=(0.0603,14.1817))$ and $(\mathbf{v i})$ solution point $(\mathrm{h})((P, Q)=(0.0600$, 14.1859)) at $R=0.3$.

(i)

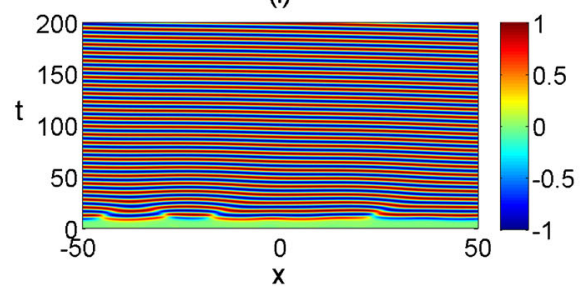

(iii)

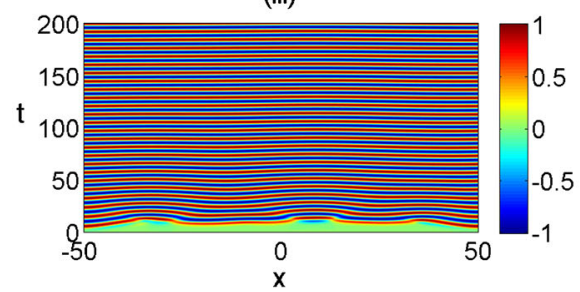

(ii)

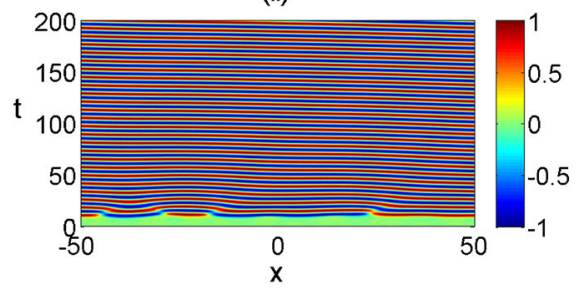

(iv)

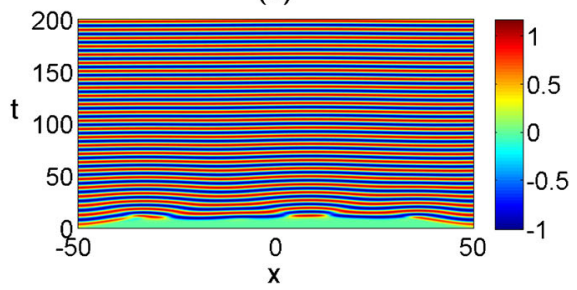

Figure 7. Numerically obtained spatiotemporal evolution of field envelope $\psi$ with respect to spatial coordinate $x$ and time t. (i) $\operatorname{Re}(\psi)$ for point (a), (ii) $\operatorname{Im}(\psi)$ for point (a), (iii) $\operatorname{Re}(\psi)$ for point (b) and (iv) $\operatorname{Im}(\psi)$ for point (b), points (a) and (b) are marked in figure 4. Colour bar for each plot is represented besides each plot. Red colour represents crest and blue colour represents trough of the oscillating wave.

width of the pulse can be calculated using the relation given by eq. (10) in ref. [50]. We have extensively investigated the solutions with different $R$ values. A large family of solutions is obtained. The solution set obtained for different $R$ values almost show a similar trend. The solution point lying on the $x$-axis in figure 4 generates the fundamental shape, here, purely Gaussian for this system, followed by flat-top or nearly flat-top profiles. As the value of $P$ decreases and $Q$ increases, the double hump starts forming in the profile with increasing depth, thus generating the family of cosh-Gaussian solutions. In a nutshell, it can be said that, the system is highly tunable within the range of the choice of parameters.
The evolution of TW solution has been studied by solving eq. (1) numerically. The split-step Fourier method [49], pseudospectral method and ETD2 exponential time-stepping method [53] have been applied to the system as referred in ref. [54]. Cosh-Gaussian function given by eq. (33) is considered as the initial profile of the TW. The spatio-temporal profile of the real and imaginary parts of field envelope $\psi$ corresponding to solution point (a) in figure 4 is presented in figures $7 \mathrm{i}$ and $7 \mathrm{ii}$ respectively, while those profiles corresponding to solution point (b) are portrayed in figures 7iii and 7iv respectively. Along with initial TW condition, slight noise of the order $10^{-3}$ is introduced in the system. Slightly disturbed initial oscillations of 


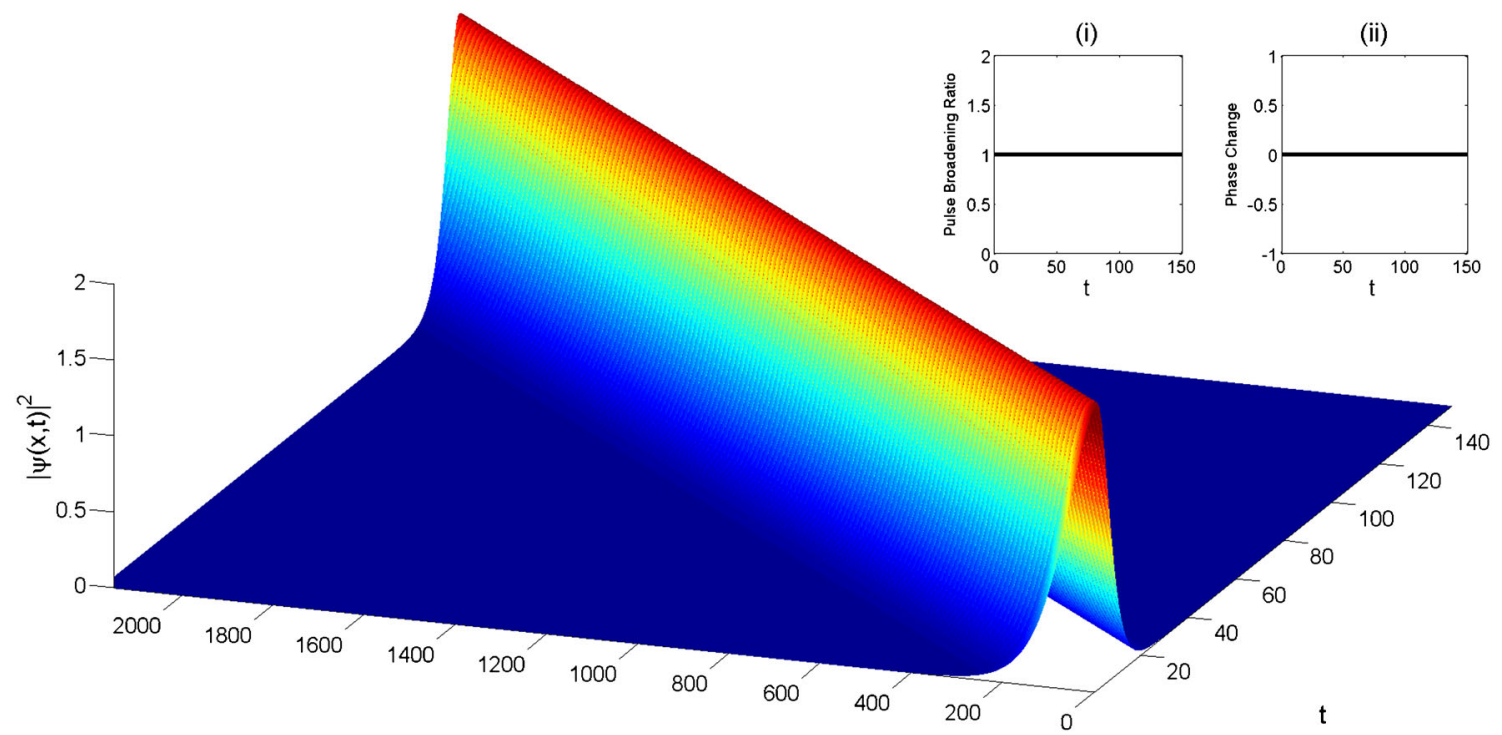

Figure 8. Numerically obtained spatiotemporal evolution of field envelope for sech input profile. Inset plot (i) corresponds to pulse broadening ratio with respect to time $t$ and (ii) corresponds to phase change countered by the field envelope with respect to time $t$.

the real and imaginary parts in the plots of wave envelope eventually become periodic, thus presenting the robustness of the system. The typical oscillatory nature of the real and imaginary parts is evident in the figures. We numerically solve the CGLE for other solution points (c)-(i) in figure 4. The oscillatory behaviour of the real and imaginary parts of $\psi$, similar to that shown in figure 7, is observed for each point.

Split-step Fourier method (SSFM) is applied to the system described by eq. (1) to study the TW solution.
Stable propagation of a TW is clearly shown in figure 8 for sech and, figures 9 and 10 for cosh-Gaussian field profiles, respectively. Figures 9 and 10 represent different profiles of cosh-Gaussian envelope obtained by direct numerical solution of eq. (1) with parameters corresponding to the analytically found solution points in figure 4. Figure 9i represents the stable propagation of fundamental Gaussian profile, followed by the flattop profile represented in figure 9iii. As $P$ decreases and cosh parameter $Q$ increases, the double hump
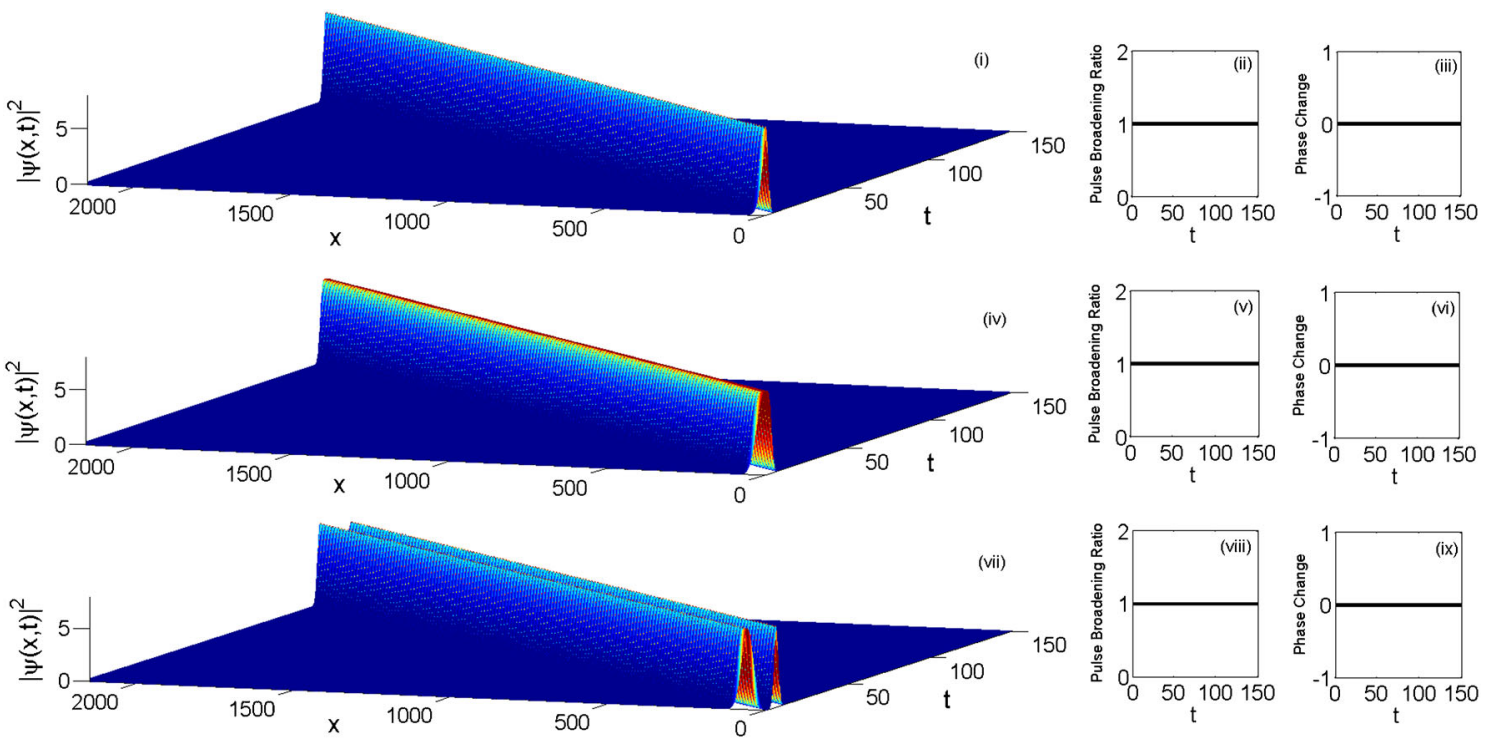

Figure 9. Numerically obtained spatiotemporal evolution of cosh-Gaussian field profile $|\psi(x, t)|^{2}$ for points: (i) solution point (a), (iv) solution point (b), (vii) solution point (c), marked in figure 4. Corresponding pulse broadening ratio and phase change countered by the field envelope with respect to time $t$ are shown in figures (ii) and (iii), (v) and (vi), and (viii) and (ix), respectively. 

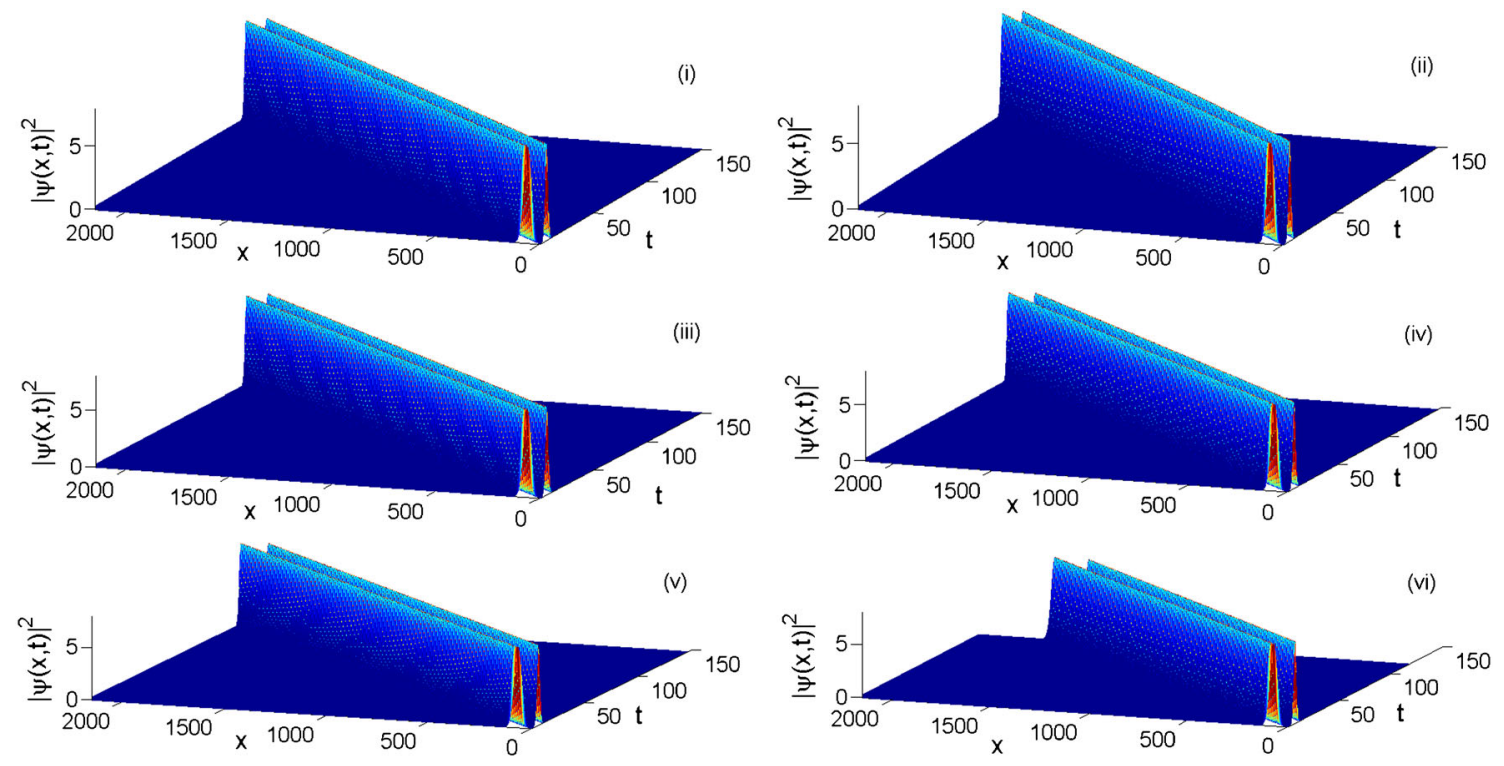

Figure 10. Numerically obtained spatiotemporal evolution of cosh-Gaussian field profile $|\psi(x, t)|^{2}$ for solution points (i) (d), (ii) (e), (iii) (f), (iv) (g), (v) (h) and (vi) (j), marked in figure 4.

starts forming in the peak. This nature of the profile is evident in figures 9vii and 10i-vi. In dissipative systems, diffraction as well as nonlinearity play important roles. The phenomenon of diffraction is mainly responsible for the pulse broadening and it can be detrimental in the systems dealing with the TW. For each solution point, pulse broadening ratio, i.e., the ratio of fullwidth at half-maxima of the input and output pulses, has been studied with respect to time. As expected, it is observed that for each case the pulse broadening ratio comes out to be unity for the total travelling time. The diffraction effect is completely balanced by the nonlinearity present in the system. Hence, the system under consideration does not undergo pulse broadening, thus, leading towards stability and robustness of the system. The other major aspect to countercheck the stability of the system is to study the phase instabilities witnessed by the system. Although phase changes insignificantly affect the pulse spectrum, but can alter the pulse shape [49], it becomes important to study the phase instabilities witnessed by the system. In the analytical study, it was considered that the system is stable against any phase changes. The numerical study of the system determined that the system has not undergone any significant phase change throughout the considered travel time. For each possible case, pulse broadening ratio and phase change are studied. Inset (i) of figures 8, 9ii, $9 \mathrm{v}$ and 9 viii represent the pulse broadening ratio and inset (ii) of figure 8, 9iii, 9vi and 9ix represent phase change with respect to time. Analytical and numerical results are found to be in impeccable conjugation with each other. Thus the numerical results qualitatively assert analytically obtained existence points of cosh-Gaussian TW solutions.

\section{Conclusion}

TW solutions of commonly used sech-type and notso-explored cosh-Gaussian type are derived for a dissipative semiconductor laser cavity. The cosh-Gaussian solution is found to be more generic as it can yield different TW profiles including the standard sech and Gaussian. A wide family of solutions with varying profile shapes are obtained. The outcome of this investigation has potential application in mode shaping, pattern formation and switching. Also, the wide parametric range will be beneficial for experimental investigations of such systems. Analytical results thus obtained open an exciting opportunity to generate family of TW profiles from a single solution set of parametric values. This can have vast applicability in different physical systems. Analytical results can build experimental basis of the systems described by eq. (1). The numerical simulation of eq. (1) is presented, which qualitatively supports the analytical results. Finally, the output of the present investigation can be used to find complicated localized pattern and CS in VCSEL.

\section{Acknowledgement}

S Jana would like to acknowledge the financial support of UGC through UGC-BSR research start-up grant, 
No. F. 20-1/2012 (BSR)/20-13 (12)/2012(BSR). B Kaur would like to acknowledge the financial support of UGC through UGC-BSR Research Fellowships in Sciences, F.4-1/2006(BSR)/7-304/2010(BSR).

\section{References}

[1] N N Akhmediev and A Ankiewicz, Solitons: Nonlinear pulses and beams (Chapman and Hall, London, 1997)

[2] N B Abraham and W J Firth, J. Opt. Soc. Am. B 7, 951 (1990)

[3] T Ackemann and W Lange, Appl. Phys. B 72, 21 (2001)

[4] F T Arecchi, S Boccaletti and P L Ramazza, Phys. Rep. 318, 1 (1999)

[5] J Ross, S C Muller and C Vidal, Science 240, 460 (1999)

[6] A C Newell, Solitons in mathematics and physics (SIAM, Philadelphia, USA, 1985)

[7] J D Murray, Mathematical biology: Spatial models and biomedical applications, 3rd edn (Springer, Berlin, 2007)

[8] N N Akhmediev and A Ankiewicz, Dissipative solitons: Lecture notes in physics (Springer, Berlin, 2005) Vol. 661

[9] T Ackemann, W J Firth and G L Oppo, Adv. At., Mol. and Opt. Phys. 57, 323 (2009)

[10] K Iga, IEEE J. Sel. Top. Quant. Electron. 6, 1201 (2000)

[11] M van-Hecke, Phys. Rev. Lett. 80, 1896 (1998)

[12] W van-Saarloos, The complex Ginzburg-Landau equation for beginners, Spatio-temporal patterns in nonequilibrium complex systems: Proceedings of the Santa Fe workshop edited by P E Cladis and P Palffy-Muhoray (Addison-Wesley, Chicago, 1994) pp. 19-31

[13] G Dangelmayr and L Kramer, Mathematical tools for pattern formation, Evolution of spontaneous structures in dissipative continuous systems edited by $\mathrm{F} \mathrm{H}$ Busse and S C Mueller (Springer, New York, 1998) pp. 1-85

[14] A Doelman, J. Nonlin. Sci. 3, 225 (1993)

[15] H Chate, Nonlinearity 7, 185 (1994)

[16] P V Paulau, A J Scroggie, A Naumenko, T Ackemann, N A Loiko and W J Firth, Phys. Rev. E 75, 056208(1) (2007)

[17] K A Montgomery and M Silber, Nonlinearity 17, 2225 (2004)

[18] J Xiao, G Hu, J Yang and J Gao, Phys. Rev. Lett. 81, 5552 (1998)

[19] Y Kuramoto and T Tsuzuki, Prog. Theor. Phys. 55, 356 (1976)

[20] I S Aranson and L Kramer, Rev. Mod. Phys. 74, 99 (2002)

[21] W van-Saarloos and P C Hohenberg, Physica D 56, 303 (1992)

[22] J D Anderson, R A Ryan, M Wu and L D Carr, New J. Phys. 16, 023025(1) (2014)

[23] Y Tanguy, T Ackemann, W J Firth and R Jager, Phys. Rev. Lett. 100, 013907(1) (2008)
[24] N Radwell and T Ackemann, IEEE J. Quant. Electron. 45, 1388 (2009)

[25] A J Scroggie, W J Firth and G L Oppo, Phys. Rev. A 80, 013829(1) (2009)

[26] P V Paulau, D Gomila, T Ackemann, N A Loiko and W J Firth, Phys. Rev. E 78, 016212(1) (2008)

[27] P Zhong, R Yang and G Yang, Phys. Lett. A 373, 19 (2008)

[28] H Cong, J Liu and X Yuan, J. Math. Phys. 50, 063516(1) (2009)

[29] R Deissler and H R Brand, Phys. Rev. Lett. 72, 478 (1992)

[30] W J Firth and P V Paulau, Eur. Phys. J. D 59, 13 (2010)

[31] B A Malomed and A A Nepomnyashchy, Phys. Rev. A 42, 6009 (1990)

[32] V Skarka, N B Aleksic, H Leblond, B A Malomed and D Mihalache, Phys. Rev. Lett. 105, 213901(1) (2010)

[33] R Goh and A Scheel, J. Nonlinear Sci. 24, 117 (2014)

[34] A V Porubov and M G Velarde, J. Math. Phys. 40, 884 (1999)

[35] L I U Cheng-Shi, Commun. Theory. Phys. 43, 787 (2005)

[36] R Conte and M Musette, Physica D 69, 1 (1993)

[37] K Nozaki and N Bekki, J. Phys. Soc. Jpn 53, 1581 (1984)

[38] L I Hua-Mei, L I N Ji and X U You-Sheng, Commun. Theor. Phys. 44, 79 (2005)

[39] V Skarka and N B Aleksic, Phys. Rev. Lett. 96, 013903(1) (2006)

[40] W Bao, Q Du and Y Zhang, SIAM J. Appl. Math. 67, 1740 (2007)

[41] J H He, Int. J. Mod. Phys. B 20, 1141 (2006)

[42] H K Khalil, Nonlinear systems 3rd edn (Prentice Hall, USA, 2002)

[43] D Puzyrev, S Yanchuk, A G Vladimirov and S V Gurevich, SIAM J. Appl. Dyn. Syst. 13, 986 (2014)

[44] B Janiaud, A Pumir, D Bensimon and V Choquette, Physica D 55, 269 (1992)

[45] A N W Hone, Physica D 205, 292 (2005)

[46] V García-Morales and K Krischer, Contemp. Phys. 53, 79 (2012)

[47] S Liu, S Liu, Z Fu and Q Zhao, Chaos, Solitons and Fractals 13, 1377 (2002)

[48] S Charkrit, Proceedings of World Congress of Engineering (London, UK, July 2013) pp. 156-160

[49] G P Agrawal, Nonlinear fiber optics, 4th edn (Academic Press, San Diego, USA, 2001)

[50] S Konar and S Jana, Opt. Commun. 236, 7 (2004)

[51] A Biswas, PIER 96, 1 (2009)

[52] S Jana and S Konar, Opt. Commun. 267, 24 (2006)

[53] S M Cox and P C Matthews, J. Comput. Phys. 176, 430 (2002)

[54] D M Winterbottom, Pattern formation with a conservation law, Ph.D. thesis (The University of Nottingham, England, 2006) 